\title{
The effectiveness of ultrasound in the diagnosis of bladder tumours at the Muhimbili National Hospital, Dar es Salaam, Tanzania
}

\author{
MASUMBUKO Y. MWASHAMBWA ${ }^{1 *}$ and SYDNEY C. YONGOLO ${ }^{2}$ \\ ${ }^{1}$ Department of Surgery and Maternal Health, the University of Dodoma, P.O. Box 259, Dodoma, Tanzania \\ ${ }^{2}$ Department of Surgery; Muhimbili University of Health and Allied Sciences, Dar es Salaam, Tanzania
}

\begin{abstract}
The importance of an ultrasound in diagnosis of bladder tumours has been investigated by different authors. Some have questioned its effectiveness while others have considered the technique to be an important tool in the initial evaluation of bladder tumours. This study was carried out to establish the effectiveness of the ultrasound in diagnosis of bladder tumours at the Muhimbili National Hospital in Dar es Salaam, Tanzania. Clinical indications and ultrasound findings were recorded. Cystoscopy was done and findings recorded on a preformed questionnaire. The ultrasound findings were compared to cystoscopy findings and the sensitivity, negativity, positive predictive value and negative predictive value determined. A total of 110 patients were recruited in this study and the male to female ratio was 2:1. The commonest (37\%) age group was $41-60$ years. The most common clinical indication overall was haematuria in $37 \%$ of all cases. In males, bladder outlet obstruction due to stricture was the commonest indication (31\%). Out of 110 patients scheduled for cystoscopy, 71 had ultrasound done preoperatively. In these patients $70 \%$ had some form of abnormal ultrasound findings. The sensitivity, specificity, positive predictive value, and the negative predictive value (NPV) of ultrasound in detection of bladder tumour were $83 \%, 93 \%, 89 \%$ and $89 \%$, respectively. In conclusion, ultrasound is an effective method for evaluating patients presenting with haematuria or suspected to have bladder tumours. It is cheap, available, affordable and non-invasive; has a high sensitivity, and therefore it can also be useful in the follow-up of patient with bladder cancer.
\end{abstract}

Key words: haematuria, bladder tumours, ultrasound, cystoscopy, urinary bladder, Tanzania

\section{Introduction}

Bladder tumours are very common in both developed and developing countries. In the western world, transitional cell carcinoma (TCC) is the commonest, while in developing countries squamous cell carcinoma (SCC) is the most prevalent especially in areas where schistosomiasis is endemic (Gary, 2004). It has been showed that $80-90 \%$ of patients with bladder tumours, present with painless haematuria, particularly in people with TCC (Gary, 2004). Patients with SCC usually present with features of cystitis and painful micturition; haematuria comes in at later stages of the illness (Aboud, 1999).

In an ideal setting the diagnosis of bladder cancer involves the use of cytology, the technique that detects malignant cells. In advanced settings this may be supplemented by the detection of tumour makers in the urine (Pode et al., 1986). The ultrasound is used as an initial test prior to cystoscopy. Ultrasound can predict the nature of the lesion(s) and has many advantages such as easily available, non-invasive, non-ionizing, and easily acceptable to patients (Fransisca et al., 2008). Cystoscopy is done to confirm the presence of the tumour but also allows biopsy and hence histological identification of the tumour. Further radiological

\footnotetext{
* Correspondence: Masumbuko Y. Mwashambwa; E-Mail: masuyt@yahoo.co.uk
} 
investigations such as computed tomography scanning (CT scan) and magnetic resonance imaging (MRI) can evaluate the extent of the disease in case of cancer (Shariat et al., 2008). In developing countries however, most of these investigations are not available and if available they are expensive and many patients cannot afford.

The effectiveness of the ultrasound in diagnosis of bladder tumours has been confirmed by some scholars (Abu-Yousef et al., 1984; Cariou et al., 1997; Fransisca et al., 2008; Konstantinos et al., 2009). However, Cariou et al. (1997) have reported it to have very low effectiveness. Despite the varying results in detection of rate of bladder tumours by using the ultrasound, it is clear that the method can be used in developing countries such as Tanzania, where there are limited diagnostic facilities. It is in this context that this study was done to assess the effectiveness of ultrasound in detection of these lesions at Muhimbili National Hospital in Tanzania.

\section{Material and methods}

\section{Study site and population}

This was a prospective hospital based descriptive study, which included all patients scheduled for cystoscopy between March 2008 and September 2008 at the Muhimbili National Hospital $(\mathrm{MNH})$ in Dar es Salaam, Tanzania. $\mathrm{MNH}$ is the main referral hospital in Tanzania and also serve as a teaching hospital for Muhimbili University of Health and Allied Sciences.

All patients presenting with symptoms such as haematuria or dysuria were included in the study. Other indications included difficulty in passing urine, persistent lower abdominal pain, and urinary incontinence. A convenient sampling technique was used, in which all patients scheduled for cystoscopy during the entire period of the study were recruited.

\section{Data collection}

The ultrasound was done by the radiologist available and in order to eliminate bias he/she was not informed about the study. Parameters from the ultrasound findings such as presence or absence of tumours, size, locations and shapes were recorded on preformed questionnaire. The findings from cystoscopy such as presence of the tumours, size, location, and shape were recorded, and results compared. Available consultant urologist (who was also part of the research team) had a final decision on cystoscopy findings. Patients with normal bladder mucosa were categorized as normal findings.

\section{Data analysis}

Data were entered into Epi-Info version 6 statistical software for data analysis. Statistical significance was determined when necessary, using Chi square. The cut-off point used for the pvalue was $5 \%$. The sensitivity was determined as the proportion of tumours detected using ultrasound divided by tumours detected at cystoscopy multiplied by 100 (i.e. $24 / 29$ x100 = 83\%). This means the probability of an ultrasound detecting a bladder tumour when the patients truly have the tumour is $83 \%$. The specificity was determined as the probability that the ultrasound will exclude patients without the bladder tumour. It was calculated from patients with no bladder tumour at ultrasound and divided by patients without bladder tumour at cystoscopy 
(i.e. $39 / 42$ x100 = approximated to $90 \%$ ). The positive predictive value (PPV) was determined as the proportion of patients with bladder tumour among all ultrasound detected tumours (i.e. $24 / 28=86 \%$ ). The negative predictive value (NPV) was determined as the proportion of patients without bladder tumour among normal bladder ultrasound findings (i.e. 39/44=88\%).

\section{Ethical considerations}

The study protocols were approved by the Muhimbili University of Health and Allied Sciences Ethical Committee. A written informed consent was obtained from all patients.

\section{Results}

A total of 110 (male $=70 \%$; female $=30 \%$ ) patients were recruited and underwent cystoscopy. Of these, 71 had prior ultrasound examinations and they were used to compare ultrasound findings and cystoscopy findings. Thirty-nine patients did not do ultrasound due to various reasons including faulty machine, power interruptions and financial constraints, and hence were not used for comparison of the two evaluation methods. Haematuria (37\%) and stricture $(23 \%)$ were the commonest indications for cystoscopy (Table 1$)$.

Table 1: Clinical indication for cystoscopy according to sex

\begin{tabular}{llll}
\hline Condition & No. of males & No. of females & No. (\%) of total patients \\
\hline Haematuria & 23 & 18 & $41(37 \%)$ \\
Stricture & 24 & 1 & $25(23 \%)$ \\
Persistent dysuria & 12 & 3 & $15(14 \%)$ \\
Lower abdominal pain & 6 & 2 & $8(7 \%)$ \\
Incontinence & 3 & 4 & $7(6 \%)$ \\
Other indications & 9 & 5 & $14(13 \%)$ \\
Total & 77 & 33 & 110 \\
\hline
\end{tabular}

The commonest pathological ultrasound finding was bladder tumour (39\%). Other pathological findings were included presence of thickened or calcific bladder mucosa (13\%) hydronephrosis (7\%) (Table 2). Patients with normal bladder mucosa or ureteric orifices were categorized as normal findings.

Table 2: Ultrasonographic findings

\begin{tabular}{lcl}
\hline Condition & Frequency & Percent \\
\hline Normal bladder findings & 22 & $31 \%$ \\
Bladder mass & 28 & $39 \%$ \\
Hydroureter/nephrosis & 5 & $7 \%$ \\
Bladder calculi & 2 & $3 \%$ \\
Thickening/Calcification (of bladder wall) & 9 & $13 \%$ \\
Bladder wall diverticulum & 2 & $3 \%$ \\
Inflammation of trigone & 3 & $4 \%$ \\
Total & 71 & $100 \%$ \\
\hline
\end{tabular}


Table 3: Bladder and cystoscopy findings among patients who underwent both ultrasound examination and cystoscopy

\begin{tabular}{llllll}
\hline Ultrasound & \multicolumn{5}{c}{ Cystoscopy } \\
& Normal & Bladder mass & Hyperaemia & Schistosomiasis & Total \\
\hline Normal & $10(43 \%)$ & $3(10 \%)$ & $6(50 \%)$ & 3 & $22(31 \%)$ \\
Bladder mass & $3(13 \%)$ & $24(83 \%)$ & $1(8 \%)$ & 0 & $28(39 \%)$ \\
Thickening/Calcification & $3(13 \%)$ & $1(3 \%)$ & $4(17 \%)$ & 3 & $11(13 \%)$ \\
Bladder calculi & $4(17 \%)$ & 0 & 0 & 1 & $5(3 \%)$ \\
Hydronephrosis & $3(13 \%)$ & $1(3.4 \%)$ & $1(8 \%)$ & 0 & $5(7 \%)$ \\
Total & $23(32 \%)$ & $29(41 \%)$ & $12(17 \%)$ & $7(4 \%)$ & 71 \\
\hline
\end{tabular}

Of 29 patients who actually had bladder masses at cystoscopy, the ultrasound had picked 24 $(83 \%)$, the ultrasound had missed 5; in 3 patients the bladder wall was observed as normal, in one patient bladder mass was observed as a thickening/calcified bladder wall and in the last patient only hydronephrosis was observed (Table 3). In 4 patients the ultrasound detected bladder mass while they didn't have the mass. The efficacy of an ultrasound in detection of bladder cancer measured in terms of sensitivity, specificity, positive predictive value (PPV) and negative predictive value (NPV) was found to be high, that is $83 \%, 90 \%, 86 \%$ and $88 \%$, respectively (Table 4). Other indications included vesico-cutaneous fistula, ureteric stent removal, and benign prostatic hyperplasia.

Table 4: Accuracy of ultrasound in diagnosis of bladder tumours

\begin{tabular}{llll}
\hline Ultrasound & $\begin{array}{l}\text { Cystoscopic } \\
\text { Bladder tumour }\end{array}$ & No bladder tumour & Total \\
\hline Bladder tumour & 24 & 4 & 28 \\
& $\begin{array}{l}\text { (Detected by both ultrasound } \\
\text { and cystoscopy) }\end{array}$ & $\begin{array}{l}\text { (Detected by ultrasound } \\
\text { but not by cystoscopy) }\end{array}$ & $\begin{array}{c}\text { (Total detected by } \\
\text { ultrasound) }\end{array}$ \\
No bladder & 5 & 38 & 43 \\
tumour & (Detected by cystoscopy, & $\begin{array}{l}\text { (Ultrasound and } \\
\text { cystoscopy negative } \\
\text { missed by ultrasound) }\end{array}$ & $\begin{array}{l}\text { (Ultrasound negative } \\
\text { findings) }\end{array}$ \\
& $\begin{array}{l}\text { findings) } \\
\text { Total }\end{array}$ & $\begin{array}{l}42 \\
\text { (cystoscopy negative } \\
\text { findings) }\end{array}$ & 71 \\
& (Total detected by & & \\
\hline
\end{tabular}

\section{Discussion}

This study was done to investigate the effectiveness of an ultrasound in diagnosis of bladder tumours. The commonest clinical indications for cystoscopy were haematuria and stricture. This trend of indications concurs with other studies (Fransisca et al., 2008 \& Olu-Eddo, 2006). Unlike in our study, in developed countries post bladder tumour resection cystoscopic surveillance is a very common indication for cystoscopy (Fransisca et al., 2008). This is attributed to the fact that, most of bladder cancer in developing countries, and particularly in Africa is of squamous cell origin (Aboud, 1999; Garry, 2004). Most of these types of cancers are usually advanced at the time of diagnosis and therefore are treated with cystectomy than 
endoscopic resection that warrants post resection cystoscopy surveillance. On the other hand, transitional cell bladder cancers which are common in developed countries (Garry, 2004) are often treated by endoscopic resection and hence the need for post resection cystoscopy surveillance.

It has been pointed out earlier that, diagnosis of bladder tumours in developing countries is challenging, because of lack of modern medical equipment in most hospitals. However, cystoscopy and ultrasound are assumed to be available in most tertiary hospitals in most developing countries. The two techniques can be used effectively for diagnosis of these tumours. Cystoscopy is the gold standard and very important diagnostic tool for evaluation of bladder cancer (Cariou et al., 1997; Fenlon et al., 1997; Amling, 2001; Fransisca et al., 2008). The ultrasound as previously stated, is cheap, available, non-ionizing, and non-invasive. However, technical and scientific advances in radiology have increased the diagnostic accuracy of ultrasound in evaluation of bladder tumours. Studies have reported an increase in detection rate of ultrasound for bladder cancers from $82 \%$ to $95 \%$ (Abu-Yousef et al., 1984; Ozden et al., 2007).

In the past the efficacy of an ultrasound in diagnosis and staging of bladder tumours was questioned by some authors (Cariou et al., 1997; Kundra \& Silverman, 2003), due to low sensitivity and specificity. However, technical and scientific advances in radiology have increased the diagnostic accuracy of ultrasound in evaluation of bladder tumours. Studies have reported an increase in detection rate of ultrasound for bladder cancers from $82 \%$ to $95 \%$ (AbuYousef et al., 1984; Ozden et al., 2007). The high sensitivity, specificity and predictive values of ultrasound in the diagnosis of the bladder tumours emphasizes that the technique can be used for diagnosis of bladder tumours in our settings. This finding is similar to observations reported by Abu- Yousef et al. (1984) and Fransisca et al. (2008). Lower sensitivity (50\%) of ultrasound has been reported in a study by Cariou et al. (1997). One of the possible explanations for the high detection rate in our study is likely to be due to the fact that, most of the patients present very late with advanced tumours that can easily be detected by an ultrasound. It is also known that haematuria is a late symptom in schistosomal related bladder tumours (Aboud, 1999). In addition, the fact that the study was done at a tertiary hospital which has adequate number of competent radiologists and that most of the patients had a referral diagnosis could have contributed to the high detection rate observed.

Although there is remarkable improvement in the ability of ultrasound to detect bladder tumours, some of the pitfalls of ultrasonographic tests for evaluation of the bladder tumours should be considered. Smaller lesions $(<0.5 \mathrm{~cm})$ and lesions located in the dome or bladder neck are more difficult to visualize using an ultrasound. Tumour configuration is also an important factor; flat lesions are almost certainly harder to detect than polypoid ones (Ozden et al., 2007). Other factors include obesity, degree of bladder distention and also the experience and skills of the performer (Abu-Yousef et al., 1984). In this study the ultrasound missed 5 patients (false negative), who actually were found to have bladder cancer at cystoscopy. Factors described above are likely to have contributed to the false negatives observed.

In conclusion, an ultrasound is an effective method for evaluation of patients presenting with haematuria or suspected to have bladder tumours. It can be used as a first line investigation in evaluation of these patients, as it is cheap, available, affordable and non- 
invasive, particularly in developing countries where there are limited facilities. It can help to shorten the duration of evaluation of patients with haematuria, but also given the high sensitivity, an ultrasound can be safely considered in post resection follow up to evaluate future recurrences.

\section{References}

Aboud, M.M. (1999) Bladder cancer. In: Textbook of Urology and Nephrology in Africa. J.L. Eschem (ed.). Pennyslvania, USA; pp 176-180.

Abu-Yousef, M., Narayana, A., Franken Jr., E. \& Brown, R. (1984) Urinary bladder tumors studied by cystosonography. Part 1: Detection. Radiology 153, 223-226.

Amling, C.L. (2001) Diagnosis and management of superficial bladder cancer. Current Ploblems in Cancer 25, 219-278.

Cariou, G., Maaraoui, N. \& Cortesse, A. (1997) Can the combination of bladder ultrasonography and urinary cytodiagnosis replace cystoscopy in the diagnosis and follow up of tumors of the bladder? Progres en urologie 7, 51-55.

Fenlon, H., Bell, T., Ahari, H. \& Hussain, S. (1997) Virtual cystoscopy: early clinical experience. Radiology 250, 272-274.

Francica, B., Bellini, S.A., Scarano, F., Miragliuolo, A., De Marino, F.A. \& Maniscalco, M. (2008) Correlation of transabdominal sonographic and cystoscopic findings in the diagnosis of focal abnormalities of the urinary bladder wall: A prospective study. Journal of Ultrasound Medicine 27, 887-894.

Garry, D.S., (2004) Bladder Cancer. Available at http://www.emedicine.com. Accessed on $7^{\text {th }}$ March 2008.

Groeneveld, A.E. (2003) Bladder cancer in various population groups in the greater Durban area of Kwa Zulu-Natal, South Africa. British Journal of Urology 78, 205-208.

Konstantinos, S., Ioannis, P., Stefanos, D., Grigoris, Z. \& Konstantinos, P. (2009) Accuracy of ultrasonography in the diagnosis of superficial bladder tumors in patients presenting with hematuria. Annals of Saudi Medicine 29, 134-137.

Kundra, V. \& Silverman, P.M. (2003) Imaging in oncology from the University of Texas M.D Anderson Cancer Center: imaging in the diagnosis, staging, and follow-up of cancer of the urinary bladder. American Journal of Roentgenology 180, 1045-1054.

Malone, P.R., Weston-Underwood, J., Aron, P.M., Wilkinson, K.W., Joseph, A.E. \& Riddle, P.R. (1986) The use of transabdominal ultrasound in the detection of early bladder tumours. British Journal of Urology 58, 520-522.

Olu-Eddo, A.N. (2006) Bladder tumor in Benin City: A 15 years histopathological study. Nigerian Journal of Health and Biomedical Sciences 5, 105-109.

Ozden, E., Turgut, A.T., Turkolmez, K., Resorlu, B. \& Safak, M. (2007) Effect of bladder carcinoma location on detection rates by ultrasonography and computed tomography. Urology 69, 889-892.

Pode, D., Alon, Y., Horowitz, A.T., Vlodavsky, I. \& Biran, S. (1986) The mechanism of human bladder tumor implantation in an in vitro model. Journal of Urology 136, 482. 
Shariat, S.F., Karam, J.A., Lerner, S.P. (2008) Molecular markers in bladder cancer. Current Opinion in Urology 18, 1-8. 